\title{
Chemo-Preventive Functions of Grape Seed Proanthocyanidin Extract against UV-Induced Intracellular Oxidative Stress and Tyrosinase Activity
}

\author{
Lei Shi, ${ }^{*, a}$ Hua-Lin Tang ${ }^{b}$ and Su-Ling Xu \\ ${ }^{a}$ Department of Dermatology, Affiliated Hospital of Medical College, Ningbo University, \\ 315020 Ningbo, Zhejiang Province, China \\ ${ }^{b}$ Department of Orthopaedics Surgery, Affiliated Hospital of Medical College, Ningbo University, \\ 315020 Ningbo, Zhejiang Province, China
}

\begin{abstract}
Clinical studies have identified that an excessive exposure of UV light can cause oxidative stress (OS) and tyrosinase enzyme over-expression, which are associated with multiple diseases including atherosclerosis, cancers, diabetics, rheumatoid arthritis. In this study, we investigated the impact of grape seed proanthocyanidin (GSPE) on regulating OS and tyrosinase activity in human epidermal melanocytes. This study revealed that GSPE did not affect cell viability and protected cells from UV induced damage in a dose-dependent manner. 5-(-6)-Carboxy-2,7-dichlorodihydro-fluorescein diacetate staining (i.e., a fluorescence staining for intracellular (OS)) indicated that GSPE reduced OS level caused by UV exposure. A similar trend was also confirmed by flow cytometry analysis, where GSPE down-regulated OS level. Tyrosinase analysis showed that GSPE treatment decreased tyrosinase activity. Taken all data together, GSPE may restore the cellular damage caused by excessive UV-exposure and promote skin health by reducing tyrosine generation. Clinically, GSPE could be potentially utilized for improving skin health against excessive UV exposure.
\end{abstract}

Keywords: grape seed proanthocyanidin extract, UV damage, reactive oxygen species, tyrosinase

\section{Introduction}

Human skin is the first line of protection against toxicants or their metabolites, which are usually oxidants that are associated with the production of reactive oxidants or reactive oxygen species (ROS), reactive substances that are continuously generated at low levels during metabolism. ${ }^{1}$ In particular, some ROS can be rapidly converted to $\mathrm{H}_{2} \mathrm{O}_{2}$, which permeates cell membranes, promoting the formation of other oxidant species such as hypochlorous acid, aldehydes, etc. Studies have also reported that the production of these oxidants can induce acute responses to the immune cells including macrophages, neutrophils, enhancing the expression of vascular endothelial growth factor that can potentially assist wound healing in the skin. ${ }^{2}$ In clinic, the increased level of intracellular oxidative stress within human bodies is associated with multiple types of diseases including atherosclerosis, cancer, diabetics, rheumatoid

*e-mail: stonelei_970@sina.cn arthritis, myocardial infarction, etc. ${ }^{3}$ On the other hand, the exposure of skin to ionizing and UV radiations or xenobiotics/drugs can promote the production of ROS. The production of ROS during this process depends on the level of UV radiation energy, which is associated with the pathogenesis of skin disorders such as cutaneous neoplasia. ${ }^{4}$ Moreover, ROS generated by biologically relevant doses of UV is related to skin cancer progression, despite multiple factors may also be involved in this mutagenic process. ${ }^{5,6}$ Similar suggestions also come from the fact that drugs causing chronic oxidative stress contribute to the development of skin cancer in azathioprine-treated patients. ${ }^{7}$

Multiple studies ${ }^{8,9}$ reported using grape seed proanthocyanidin extract (GSPE) to inhibit oxidative stress for different purposes including cancer treatment, immune protection and skin pigment removal. In the photocarcinogenesis related study, ${ }^{10}$ GSPE was employed to supplement the diet of hairless mice at the levels of 0.2 and $0.5 \% \mathrm{~m} / \mathrm{m}$. The study illustrated that oral uptake of GSPE by the mice resulted in a less percentage of mice 
with tumors, tumor multiplicity and tumor size compared to mice with unsuplemented diet. ${ }^{10}$ Besides using GSPE against cancers, oral administration of $1 \%$ GSPE $(\mathrm{m} / \mathrm{m})$ was employed to reduce the level of UV-induced pigmentation. ${ }^{11}$ In an 8-week study, the researchers identified that GSPE feeding was comparable to the oral use of vitamin C $(\% \mathrm{~m} / \mathrm{m}) .{ }^{11}$ However, one potential issue in the high oral dose usage of GSPE was that GSPE had certain level of cytotoxicity towards certain organs including human breast, lung etc. ${ }^{8}$ GSPE was also used for radical scavenging towards free radicals generated by biological components such as superoxide anion, hydroxyl radicals and peroxyl radicals, generating superior functionalities that were comparable to vitamins $\mathrm{C}, \mathrm{E}$, and $\beta$-carotene. ${ }^{12}$ Similar results were also obtained by using GSPE against the oxidative stress caused by tobacco. ${ }^{8}$ In another study, GSPE protected against myocardial ischemia-reperfusion injury and myocardial infarction in rats, and prevented the rats from cardiotoxicity caused by the anticancer drug (i.e., doxorubicin). ${ }^{13}$ In human trials, GSPE reduced cholesterol levels significantly. ${ }^{14}$

Besides investigating the thrapeutic effects of GSPE, multiple studies ${ }^{6,8,15}$ have been trying to reveal the molecular mechanisms of GPSE in impacting the in vitro and in vivo biological functions, although the exact mechanism is still not clear. For example, in one study ${ }^{16}$ researchers found that GSPE functions through suppressing IL-17 production and enhancing Foxp3 expression (type II collagen-reactive Treg cells) in CD4+ T cells of joints and splenocytes. Addtionally, the presence of GSPE down-regulated the levels of IL-21, IL-22, IL-26 and IL-17 secretion from human CD4+ T cells in a manner that depends on STAT3 pathways. In another study, ${ }^{8}$ GSPE offered protection against oxidative stress and free radical mediated tissue injury through down-regulating bcl2 gene and the oncogene c-myc. In this work, we focused on studying the impact of GSPE to the intracellular levels of ROS and tyrosinase activity. Through this intracellular assessment, we would like to reveal how GSPE impacts oxidative stress and tyrosinase directly. Briefly, we employed GSPE to regulate the production of ROS and tyrosinase enzyme induced by UV exposure. The impact of GSPE on cellular viability and oxidative stress level was tested via fluorescence staining and flow cytometry. The influence of GSPE on tyrosinase was also assessed by comparing the activity of this enzyme under different conditions (i.e., UV-exposure, GSPE protection, etc). Together, this study illustrated that GSPE can down-regulate ROS and tyrosinase activity in the UV-exposure environment, indicating that GSPE could improve skin health in clinic.

\section{Experimental}

\section{Materials}

Grape seed proanthocyanidin extract (GSPE) was obtained from Tianjin Jianfeng Natural Product R\&D Co., Ltd. (Tianjin, China). The GSPE has $\geq 95.0 \%$ proanthocyanidins and $\geq 4.0 \%$ proanthocyanidins $\mathrm{B} 2$ as indicated by the manufacturer. Phosphate buffered saline (PBS 1x) was from VWR (Shanghai, China). 4',6-Diamidino-2-phenylindole (DAPI) was from Invitrogen (Beijing, China). RPMI (Roswell Park Memorial Institute) cell culture medium, fetal bovine serum (FBS), penicillin and streptomycin were obtained from VWR (Shanghai, China). Dimethyl sulfoxide (DMSO) was from the Sigma Chemical (Shanghai, China).

\section{Cell culture}

RPMI 1640 medium supplemented with $10 \%$ FBS, $100 \mathrm{U} \mathrm{mL}^{-1}$ penicillin and $100 \mathrm{mg} \mathrm{mL}^{-1}$ was used for cell culture. Cells were raised in $5 \% \mathrm{CO}_{2}$ at $37^{\circ} \mathrm{C}$. Cell culture medium was changed every $48 \mathrm{~h}$.

\section{UV exposure treatment}

Cells cultured in medium at $37{ }^{\circ} \mathrm{C}$ with $5 \% \mathrm{CO}_{2}$ was employed for UV test. The following UV treatment was employed for the exposure studies: an ultraviolet lamp (Model B100AP, Blak-Ray) was employed to treat the exposed cells. The lamp generated light with a wavelength of $365 \mathrm{~nm}$ and had an intensity of $6 \mathrm{~mW} \mathrm{~cm}{ }^{-2}$. A $15 \mathrm{~min}$ exposure time was used for the treatment unless explained with extra details.

\section{Viability study}

For viability study, cells were treated with different concentrations of GSPE (i.e., 3, 10, 25, and $50 \mu \mathrm{g} \mathrm{mL}^{-1}$ ) for $48 \mathrm{~h}$. Live/dead assay was used to test the cellular viability. Briefly, cells were washed twice with $1 \mathrm{~mL}$ PBS, and then treated with $100 \mu \mathrm{L}$ of dual LIVE/DEAD ${ }^{\mathrm{TM}}$ Viability (Thermofisher Scientific, Shanghai, China) for mammalian cells. The cells were kept in dark for $15 \mathrm{~min}$, and then visualized under fluorescence microscope. To test the ratio of viable cells after different treatments, cells were stained with Trypan Blue (1:1,000 in PBS) for counting. The level of viable cells was recorded by using a cell counter (NanoEnTek-E1012V-EVE automated cell counter, NanoEnTek, Inc., Seoul, Korea). 


\section{Proliferation assay}

In the proliferation study, cells at a density of $1 \times 10^{4}$ cells per well were seeded into 96 -well plates and incubated for $24 \mathrm{~h}$ before experiments. Four groups of cells were used for the study: the untreated cells were used as a control; cells treated with UV light $\left(6 \mathrm{~mW} \mathrm{~cm}^{-2}\right.$ for $\left.15 \mathrm{~min}\right)$; cells treated with GSPE $\left(50 \mu \mathrm{g} \mathrm{mL}^{-1}\right)$; and cells treated with both UV light and GSPE $\left(6 \mathrm{~mW} \mathrm{~cm}^{-2}\right.$ for $15 \mathrm{~min}$ plus $\left.50 \mu \mathrm{g} \mathrm{mL}^{-1} \mathrm{GSPE}\right)$. GSPE was added $12 \mathrm{~h}$ before UV light exposure. Cells were harvested every $12 \mathrm{~h}$ for counting.

\section{ROS assessment}

ROS detection kit (Image-iT ${ }^{\mathrm{TM}}$ LIVE Green Reactive Oxygen Species, Thermofisher, Shanghai, China) was used to measure the level of free radicals in the cells from different treatment groups. Basically, the detection kit includes 5-(-6)-carboxy-2,7-di-chlorodihydro-fluorescein diacetate (carboxyl H2 DCFDA), which is a fluorescence marker that can penetrate into live cells. Once the carboxyl H2 DCFDA was de-acetylated via free radicals, a green fluorescence could be detected under fluorescence microscope or flow cytometer.

\section{Tyrosinase activity measurement}

The level of tyrosinase activity was measured by assessing the 3,4-dihydroxy-phenylalanine (DOPA, Sigma, Shanghai, China) oxidase activity. Briefly, $5 \times 10^{6}$ cells were collected and washed with PBS twice. The cells were then collected by centrifugation $(500 \times \mathrm{g}, 5 \mathrm{~min})$. The cells were then dissolved in $1 \mathrm{~mL}$ sodium deoxycholate $(0.5 \% \mathrm{~m} / \mathrm{m})$ in distilled water and kept on ice for $15 \mathrm{~min}$.
Tyrosinase activity was assessed spectrophotometrically via measuring the oxidation of DOPA to DOPA achrome. The abosorbance at $475 \mathrm{~nm}$ was employed at $37{ }^{\circ} \mathrm{C}$ for assessment. The measurement was expressed as a relative percentage of control.

\section{Flow cytometry}

Flow cytometry analysis was done by using an Accuri C6 cytometer (BD Bioscience Inc., CA, USA) The cytometer has an air-cooled laser $(20 \mathrm{~mW})$ with wavelengths of 488 and $640 \mathrm{~nm}$. The cells were treated with ROS detection kit, followed by PBS washing twice. Cells were analyzed under flow cytometry, and those cells with no treatment were employed as control.

\section{Statistical analysis}

Data was analyzed with standard statistical analysis comparing the treated groups with control group (untreated group). Analysis of variance (ANOVA) test was used to illustrate the significant difference between groups by using Student's $t$-test. All values were reported as mean \pm standard deviation (SD) and $p<0.05$ was used as a measure of significance.

\section{Results and Discussion}

The study started by testing the impact of GSPE on cell viability. GSPE at different concentrations (3, 10, 25 , and $50 \mu \mathrm{g} \mathrm{mL}^{-1}$ ) were employed for the study. Cells with no treatment (i.e., untreated) were used as control (Figure 1a). The test illustrated that GSPE under different concentrations had similar viability: the cells treated
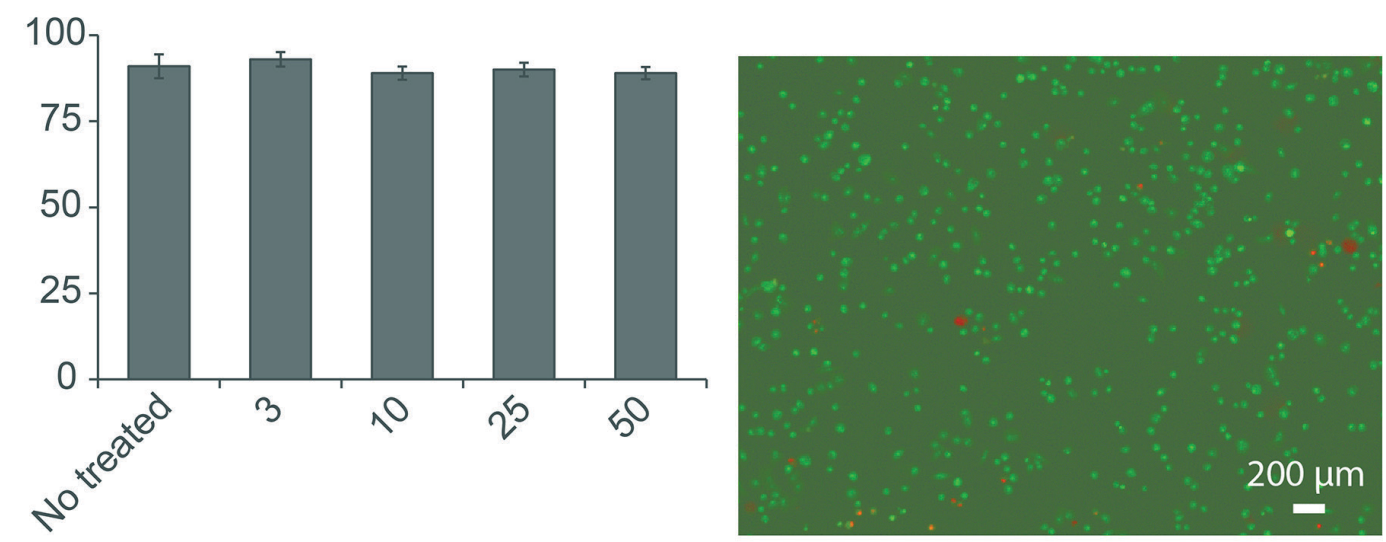

Figure 1. Impact of GSPE on cell viability. (a) GSPE at different concentrations did not affect cell viability. A range of GSPE concentrations were used to test its impact on cell viability (i.e., 3, 10, 25, $50 \mu \mathrm{g} \mathrm{mL}^{-1}$ ). Cells with no GSPE treatment were used as control (i.e., CTRL); (b) fluorescence staining of cells that were treated with $25 \mu \mathrm{g} \mathrm{mL}{ }^{-1}$ GSPE for $24 \mathrm{~h}$. Cells were washed twice with PBS and then detached from the Petri dish with trypsin. Cell viability was tested with live/dead assay, and then washed with PBS to remove the free dye. The green fluorescence indicated the viable cells $(p>0.05$ among all groups). 
with $3,10,25$, and $50 \mu \mathrm{g} \mathrm{mL} \mathrm{m}^{-1} \mathrm{GSPE}$ had $93.0 \pm 2.1 \%$, $89.1 \pm 1.9 \%, 91.2 \pm 3.5 \%$, and $90.2 \pm 2.0 \%$ viable cells, respectively, compared to $91.2 \pm 3.5 \%$ live cells in the untreated samples ( $p>0.05$ among all groups, Figure 1a). This data indicated that GSPE did not affect cell viability. This trend was also confirmed by live/dead cell staining under fluorescence microscope: the cells were treated with $25 \mu \mathrm{g} \mathrm{mL}^{-1} \mathrm{GSPE}$ for $48 \mathrm{~h}$, and the cells were still alive as indicated in green color (Figure 1b); as a contrast, a very limited number of dead cells were observed under fluorescence microscope, as indicated in red color in Figure $1 b$, which happened naturally since cells will die in their cellular cycle. Together, the viability study showed that GSPE was not toxic to cells.

Following the viability study, we studied the chemoprotective effect of GSPE to cell apoptosis caused by UV light exposure. For this study, UV light $\left(6 \mathrm{~mW} \mathrm{~cm}^{-2}\right.$ for $15 \mathrm{~min}$ ) was used to treat the cells. With no GSPE treatment, only $59 \pm 4.5 \%$ cells were viable (Figure 2 ). By pre-treating the cells with different concentration of GSPE, the ratio of live cells upon UV light treatment increased. Briefly, a treatment of cells with $3,10,25$, and $50 \mu \mathrm{g} \mathrm{mL}^{-1}$ GSPE before the UV-light exposure led to $62.2 \pm 6.1 \%$, $73.1 \pm 3.9 \%, 75.0 \pm 5.2 \%$, and $83.5 \pm 4.8 \%$ viable cells, respectively (Figure 2). The increased ratio of viable cells by GSPE pre-treatment indicated that GSPE can protect cells from UV-induced damage.

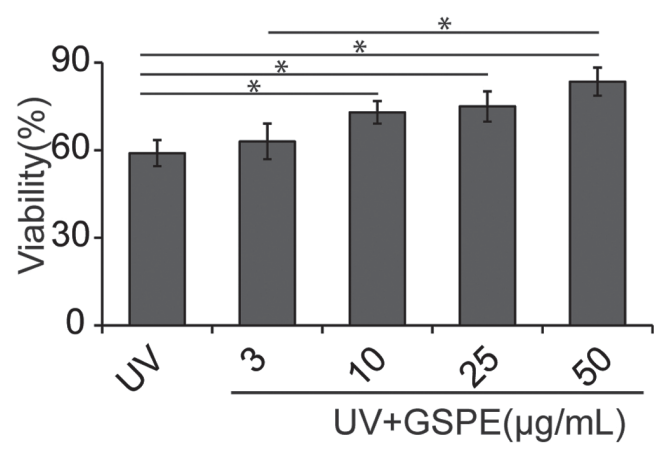

Figure 2. Chemo-protective effect of GSPE against UV exposure. Cells were treated with different concentrations of GSPE (i.e., 3, 10, 25, $\left.50 \mu \mathrm{g} \mathrm{mL}{ }^{-1}\right)$ for $12 \mathrm{~h}$, and then treated with UV light $\left(6 \mathrm{~mW} \mathrm{~cm}^{-2}\right.$ for $15 \mathrm{~min})$. The level of viable cells was then measured to assess the chemoprotective effect of GSPE $(* p<0.05)$.

Cell proliferation is an important indicator, which shows that the cells maintain their normal functions. Thus, we assessed the impact of GSPE in cell proliferation functions. In this study, the cells were treated with $6 \mathrm{~mW} \mathrm{~cm}^{-2} \mathrm{UV}$ light for $15 \mathrm{~min}$ for every $24 \mathrm{~h}$. Cell proliferation was assessed by counting the number of cells periodically (i.e., every 12 or $24 \mathrm{~h}$ ). For cells with no treatment, there was significant progression of cells over $96 \mathrm{~h}$ of incubation (Figure 3, blue triangle line). The addition of $25 \mu \mathrm{g} \mathrm{mL} \mathrm{m}^{-1} \mathrm{GSPE}$ to cell culture medium did not affect cell progression (Figure 3, green diamond line). As a contrast, the periodical adoption of UV light on cells induced a significant damage to cell proliferation. After UV light treatment, the number of cells reduced from $2 \times 10^{5}$ to $1.5 \times 10^{5}(p<0.05$, Figure 3 , black circle line). The growth of cells was further damaged after $96 \mathrm{~h}$, reducing to $0.4 \times 10^{5}$ ( $p<0.01$ between 0 and $96 \mathrm{~h}$ ), compared to $9.3 \times 10^{5}$ and $9.0 \times 10^{5}$ cells in the samples with no treatment or treated with GSPE, respectively $(p>0.05$, Figure 3). These data indicated the periodical treatment of cells with UV light could induce significant inhibition to cell growth. Upon the use of GSPE, cell growth capacity was partially restored, with the treatment of $25 \mu \mathrm{g} \mathrm{mL}^{-1}$ GSPE, the number of cells after $24 \mathrm{~h}$ culture increased to $2.13 \times 10^{5}$, compared to $2.45 \times 10^{5}$ cells in samples with no treatment $(p<0.05)$. After $96 \mathrm{~h}$ of culture, the samples with GSPE treatment had $6.43 \times 10^{5}$ cells, compared to $9.2 \times 10^{5}$ cells in the untreated samples $(p<0.01$, Figure 3). Together, these studies indicated that GSPE can partially restore the damage that UV light imposed to cell proliferation. Compared to our study, existing studies have also illustrated the protective effect of GSPE against cellular damage. For example, Shao et al. ${ }^{17}$ reported that GSPE can partially prevent cellular damage or death caused by antimycin, which is a chemical that can induce endogenous oxidant stress. Similarly, other authors also reported that GSPE protected cultured macrophages against the oxidative stress caused by $\mathrm{H}_{2} \mathrm{O}_{2} \cdot{ }^{18}$ These studies thus supported our result, allowing us to explore the following studies.

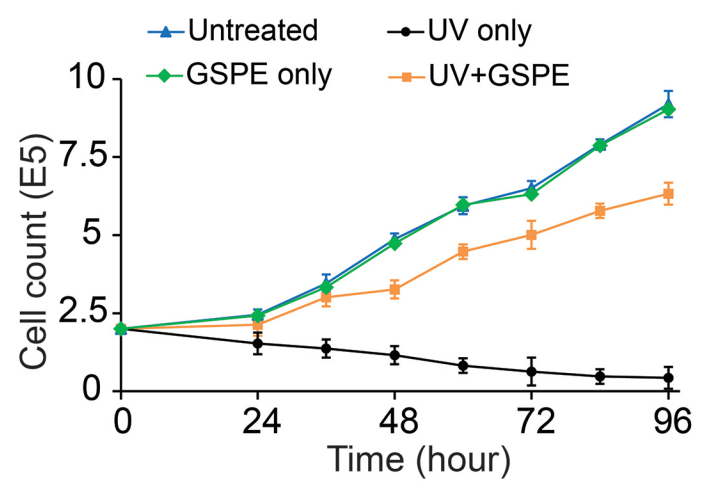

Figure 3. Impact of GSPE on cell proliferations. Four groups of cells were involved in the study: ( i) cells with no treatment (i.e., untreated); ( $\mathrm{ii}$ ) cells treated with UV light only $\left(6 \mathrm{~mW} \mathrm{~cm}^{-2}\right.$ for $\left.15 \mathrm{~min}\right)$; (iii) cells treated with GSPE $\left(25 \mu \mathrm{g} \mathrm{mL}^{-1}\right)$ only; (iv) cells treated with both UV light and GSPE ( $25 \mu \mathrm{g} \mathrm{mL}^{-1} \mathrm{GSPE}$ plus $6 \mathrm{~mW} \mathrm{~cm}^{-2}$ for $15 \mathrm{~min}$ ). The levels of viable cells were measured in the first $24 \mathrm{~h}$ after treatment and then measured every $12 \mathrm{~h}$ after the first assessment.

The following study then focused on investigating the protective mechanism of GSPE against cellular damage caused by UV light. In this work, we studied the impact 
of GSPE treatment on oxidative stress. The cells were first treated with $50 \mu \mathrm{g} \mathrm{mL}^{-1}$ GSPE for $24 \mathrm{~h}$. Cells with no GSPE treatment was used as a control. These two groups of cells were stained with carboxyl H2 DCFDA to illustrate the level of intracellular oxidative stress, followed by visualization under fluorescence microscope. For the localization of cells, cell nucleus was stained by DAPI (blue color) (Figures $4 \mathrm{a}$ and $4 \mathrm{~b}$ ). The cells under GSPE treatment had a low level of oxidative stress, as illustrated by green color within the cells (Figure 4a); as a contrast, cells with no GSPE protection showed strong green color, an indicator showing that a high level of oxidative stress was produced during UV light exposure (Figure 4b). Using flow cytometry, we quantified the level of oxidative stress within the cells. $12.7 \%$ of the GSPE-protected cells had a high level of ROS release (Figure 4c, left); as a contrast, among the cells with no GSPE protection, $53.2 \%$ cells showed a high level of oxidative stress, indicating the protective effect of GSPE against UV-induced reactive oxygen production (Figure 4c, right). This data indicated that GSPE can reduce the production of oxidative stress and partially explained why GSPE restored the damage caused by UV-light on cell growth (Figure 3). Previous studies have identified that GSPE can reduce the production of thiobarbituric acid reactive substances (TBARS, another type of oxidative substances that can be induced by cisplatin), anti-tumor drugs that kill cancer cells, but damage normal cells as well. ${ }^{19}$ Our study thus revealed another potential mechanism that might be involved in the protective functions of GSPE. However, in Figure 3, our study revealed that GSPE can help restore the cellular growth damage from UV exposure. Comparing this result with the study in Figure 4, it is possible the UV-damage restore were associated with the anti-oxidant effects of GSPE. However, considering GSPE might be involved in multiple intracellular pathways, more mechanisms studies are required to reveal the exact relation between the two results. ${ }^{9}, 16,17$
Besides studying the impact of GSPE on UV-induced ROS, we further studied how GSPE influenced the intracellular production of UV-induced tyrosinase. Figure 5 illustrated the productions of tyrosinase from cells with different treatments. The cells were treated with different doses of GSPE $\left(0,10,25\right.$ and $\left.50 \mu \mathrm{g} \mathrm{mL}^{-1}\right)$ plus UV as an irritation. Cells with no treatment was used as control (Figure 5). UV treatment led to enhancement of tyrosinase activity to a level of $257 \pm 32.1 \%$ higher than the control (i.e., untreated cells, $p<0.05$ ) (Figure 5). The use of GSPE reduced the production of tyrosinase in a dose dependent manner (Figure 5). For UV-irritated cells, the addition of 10,25 and $50 \mu \mathrm{gL}^{-1} \mathrm{GSPE}$ to the cells resulted in different relative level of tryrosinase activity of $223 \pm 11.9 \%$, $190.2 \pm 9.2 \%$ and $129 \pm 5.8 \%$, respectively, compared to the untreated cells (Figure 5). This data indicated that GSPE reduced the tyrosinase activity. The exposure of UV light to human can promote the production of melanin, which is the key substance that controls skin pigmentation and aging. ${ }^{20}$ Tyrosinase is the key and rate-limiting enzyme that is involved in melanin production. Thus, an elucidation of the effect of GSPE on tyrosinase synthesis can help us understand the protective effect of GSPE, especially in UV induced skin pigmentation and aging. ${ }^{20}$ The conclusion made in Figure 5 is also consistent with other study. For example, in one study, oligomeric proanthocyanidins, the extract grape skin seeds, were employed to reduce the over expression of tyrosinase caused by UV exposure. By using western blot, this study illustrated that oligomeric proanthocyanidins can down-regulate the synthesis of tyrosinase related protein 1 (TRP1) and 2 (TRP2), thus providing a supportive data to our study via a different characterization technique. ${ }^{15}$ Additionally, the GSPE used in our study is composed of proanthocyanidins $(\mathrm{UV}) \geq 95.0 \%$ and proanthocyanidins B2(HPLC) $\geq 4.0 \%$. Although existing studies illustrating therapeutic effects of GSPE mixture, further studies are required to illustrate the exact functions of each component. This issue requires more attention when studies involve investigating the
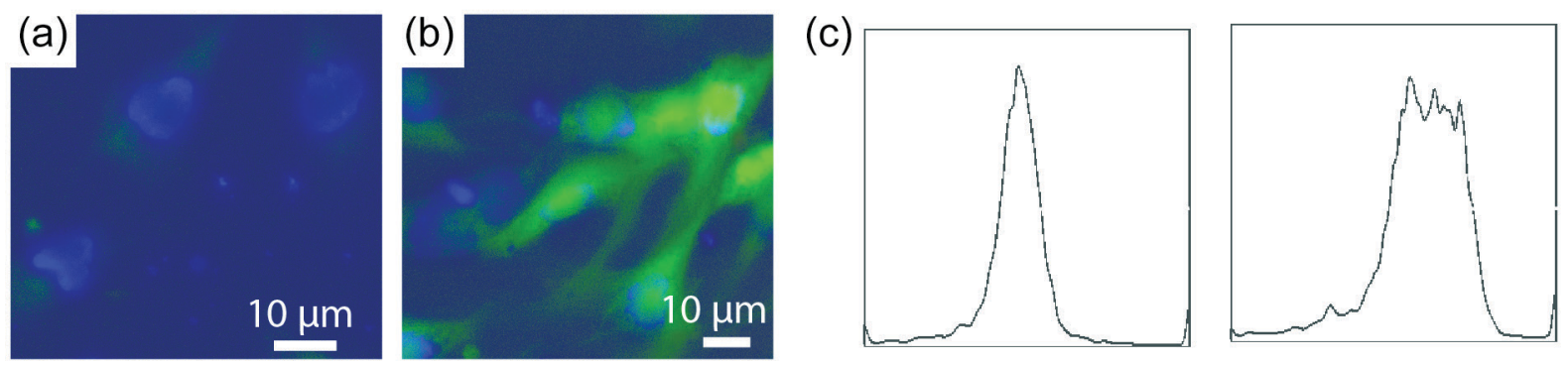

Figure 4. Carboxyl H2 DCFDA staining and flow cytometry for assessing the impact of GSPE on the UV-induced oxidative stress. (a) Carboxyl H2 DCFDA staining for measuring the level of intracellular reactive oxidative stress. Cells treated with GSPE and UV light $\left(6 \mathrm{~mW} \mathrm{~cm}^{-2}\right.$ for $\left.15 \mathrm{~min}\right)$ and (b) cells treated with UV light ( $6 \mathrm{~mW} \mathrm{~cm}^{-2}$ for $15 \mathrm{~min}$ ) but no GSPE; (c) flow cytometry for assessing the level of reactive oxidative stress in cells: (left) cells treated with GSPE and UV light; (right) cells treated with UV light only (i.e., no GSPE, $p<0.05$ between the two groups). 
fundamental mechanisms of GSPE in regulating cells or impacting the immune systems, where multiple molecular pathways might be involved.

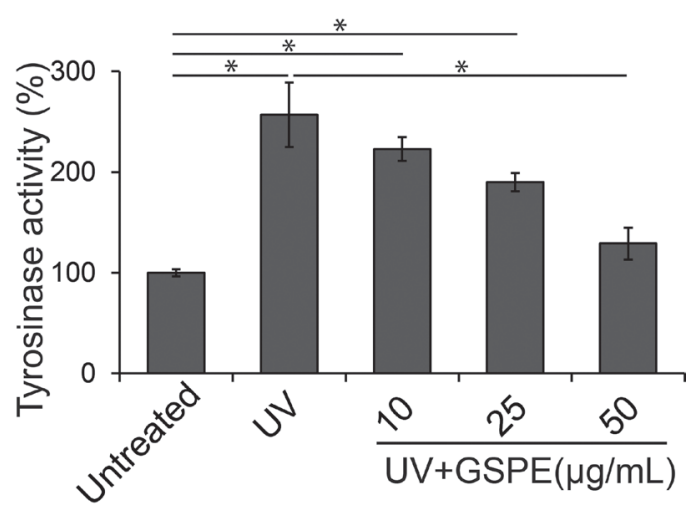

Figure 5. Impact of GSPE treatment on the production of tyrosinase induced by UV exposure. Cells were treated with different concentrations of GSPE (i.e., 10, 25, $50 \mu \mathrm{g} \mathrm{mL}^{-1}$ ) for $12 \mathrm{~h}$ and then exposed to UV light ( $6 \mathrm{~mW} \mathrm{~cm}^{-2}$ for $15 \mathrm{~min}$ ). Cells with no treatment and cells treated with UV light ( $6 \mathrm{~mW} \mathrm{~cm}{ }^{-2}$ for $15 \mathrm{~min}$, no GSPE) only were used as controls.

\section{Conclusions}

This work studied the impact of GSPE on cell viability, proliferation, UV-induced reactive oxygen stress and tryosinase production. The studies revealed that GSPE did not affect cell viability with the doses employed in this work, and GSPE can partially restore the UV-induced damage on cell proliferation. Additionally, GSPE regulated the production of oxidative stress induced by UV light exposure, thus reduced the cellular damage by decreasing the production of reactive oxygen within the cells, as confirmed by fluorescence staining and flow cytometry. Additionally, GSPE down-regulated the intracellular production of tryrosinase caused by UV-exposure. Overall, this work indicated that GSPE could be used for cellular protection against UV-induced reactive oxygen species production and tyrosinase over-expression. Clinically, the studies indicated that GSPE can be used to improve skin health by regulating these above-mentioned cellular functions.

\section{References}

1. Bickers, D. R.; Athar, M.; J. Invest. Dermatol. 2006, 126, 2565.

2. Khanna, S.; Roy, S.; Bagchi, D.; Bagchi, M.; Sen, C. K.; Free Radical Biol. Med. 2001, 31, 38.

3. Lucas, M. L.; Carraro, C. C.; Bello-Klein, A.; Kalil, A. N.; Aerts, N.; Braz. J. Cardiovasc. Surg. 2016, 31, 428; Ritchie, R. H.;
Drummond, G. R.; Sobey, C. G.; de Silva, T. M.; Kemp-Harper, B. K.; Pharmacol. Res. 2017, 116, 57; Turkmen, K.; Int. Urol. Nephrol. 2017, 49, 837; Vakonaki, E.; Tsarouhas, K.; Spandidos, D. A.; Tsatsakis, A. M.; Anatolian J. Cardiol. 2016, 16, 939.

4. Kruk, J.; Duchnik, E.; Asian Pac. J. Cancer Prev. (APJCP) 2014, 15, 561.

5. Poillet-Perez, L.; Despouy, G.; Delage-Mourroux, R.; BoyerGuittaut, M.; Redox Biol. 2015, 4, 184.

6. Kang, S. W.; Lee, S.; Lee, E. K.; Arch. Pharmacal Res. 2015 , 38,338 .

7. Ulrich, C.; Stockfleth, E.; Nephrol., Dial., Transplant. 2007, 22, 1027.

8. Bagchi, D.; Bagchi, M.; Stohs, S. J.; Das, D. K.; Ray, S. D.; Kuszynski, C. A.; Joshi, S. S.; Pruess, H. G.; Toxicology 2000, 148, 187.

9. Katiyar, S. K.; Mol. Nutr. Food Res. 2008, 52, S71.

10. Mittal, A.; Elmets, C. A.; Katiyar, S. K.; Carcinogenesis 2003, 24, 1379.

11. Yamakoshi, J.; Otsuka, F.; Sano, A.; Tokutake, S.; Saito, M.; Kikuchi, M.; Kubota, Y.; Pigm. Cell Res. 2003, 16, 629.

12. Bagchi, D.; Garg, A.; Krohn, R. L.; Bagchi, M.; Tran, M. X.; Stohs, S. J.; Res. Commun. Mol. Pathol. Pharmacol. 1997, 95, 179.

13. Sato, M.; Maulik, G.; Ray, P. S.; Bagchi, D.; Das, D. K.; J. Mol. Cell. Cardiol. 1999, 31, 1289; Ray, S. D.; Patel, D.; Wong, V.; Bagchi, D.; Res. Commun. Mol. Pathol. Pharmacol. 2000, 107, 137.

14. Preuss, H. G.; Wallerstedt, D.; Talpur, N.; Tutuncuoglu, S. O.; Echard, B.; Myers, A.; Bui, M.; Bagchi, D.; J. Med. 2000, 31, 227.

15. Zi, S. X.; Ma, H. J.; Li, Y.; Liu, W.; Yang, Q. Q.; Zhao, G.; Lian, S.; Int. J. Mol. Med. 2009, 23, 197.

16. Park, M. K.; Park, J. S.; Cho, M. L.; Oh, H. J.; Heo, Y. J.; Woo, Y. J.; Heo, Y. M.; Park, M. J.; Park, H. S.; Park, S. H.; Kim, H. Y.; Min, J. K.; Immunol. Lett. 2011, 135, 50.

17. Shao, Z. H.; Becker, L. B.; Vanden Hoek, T. L.; Schumacker, P. T.; Li, C. Q.; Zhao, D.; Wojcik, K.; Anderson, T.; Qin, Y.; Dey, L.; Yuan, C. S.; Pharmacol. Res. 2003, 47, 463.

18. Janero, D. R.; Hreniuk, D.; Sharif, H. M.; J. Cell. Physiol. 1993, 155, 494.

19. Yousef, M. I.; Saad, A. A.; El-Shennawy, L. K.; Food Chem. Toxicol. 2009, 47, 1176.

20. Bae-Harboe, Y. S. C.; Park, H. Y.; J. Invest. Dermatol. 2012, 132, 2678.

Submitted: December 9, 2017 Published online: May 22, 2018 